\title{
On time and ensemble averages in quasistationary state of low-dimensional Hamiltonian maps
}

\author{
Fulvio Baldovin \\ Centro Brasileiro de Pesquisas Físicas, Rua Xavier Sigaud 150, Urca 22290-180 \\ Rio De Janeiro - RJ, Brazil
}

\begin{abstract}
We discuss the relation between ensemble and time averages for quasistationary states of low-dimensional symplectic maps that present remarkable analogies with similar states detected in many-body long-range-interacting Hamiltonian systems.
\end{abstract}

Key words: Nonlinear dynamics, Statistical mechanics, Quasistationary states PACS: 05.10.-a, 05.20.Gc, 05.45.Ac, 05.60.Cd

\section{Introduction}

A possible approach to complex phenomena, when complete solutions are not available, is to reduce the problem into a simpler one that still retains the important features of interest, and to use the simplified version as a model that gives some insight about the original issue. Along these lines low-dimensional maps are useful tools, widely used in the literature, both to study specific behaviors of nonlinear systems with few degrees of freedom, and to understand the macroscopic properties of much larger systems. The macroscopic behavior that we are addressing in this paper is the emergence (depending on initial data) of quasistationary states (QSSs) characterized by an anomalous almost-constant value of macroscopic variables (like the average kinetic energy), before a crossover to equilibrium. These QSSs where for example displayed in the dynamics of long-range $N$-body Hamiltonian Mean Field (HMF) models where it has been shown the emergence of long-standing phases with

Email address: baldovin@cbpf.br (Fulvio Baldovin). 
non-Gaussian velocity distributions and with an anomalous value of the temperature [1], before the Boltzmann-Gibbs (BG) equilibrium is attained. The physics associated with these QSSs is extremely rich, for example various connections with nonextensive statistical mechanics [1], with aging [2] and glassy dynamics [3] have been pointed out.

Using a properly defined 'dynamical temperature' in coupled symplectic maps, it is possible to exhibit QSSs similar to those observed in the HMF model $[4,5]$. In fact, the presence of well known mechanisms (see, e.g., $[6,7]$ ) that are related to the Kolmogorov-Arnold-Moser (KAM) theory provides a possible theoretical background for the explanation of such anomalous phases. Of course, the new interest in these results stems from the possibility of better understanding the dynamical mechanisms that lead to violations of the classical BG theory, such as the one discussed in [1]. In this paper we restrict to low-dimensions, addressing the connection between ensemble and time averages. The former were already studied in [4]; the latter are obtained here following the dynamical behavior of a single trajectory, in a similar way as a thermometer time-integrates the interactions with the system to provide the output temperature.

\section{QSSs using ensemble averages}

Symplectic maps are convenient tools for the study of Hamiltonian systems, since they are the result of a Poincaré section in the phase space of a Hamiltonian system. As pointed out in [4], QSSs similar to those detected in the HMF model can be reproduced in low-dimensional symplectic maps using ensemble averages. In this section we report the main results.

In connection with the KAM theory, one of the most studied symplectic maps is the standard map [8], which is 2-dimensional. Since important qualitative changes in the topology of phase space occur when the Hamiltonian has more than two degrees of freedom (e.g., Arnold diffusion), it is also important to address this more general context by considering for example a 4-dimensional symplectic map obtained by coupling two standard maps:

$$
\begin{array}{ll}
p_{1}(t+1)=p_{1}(t)+\frac{a_{1}}{2 \pi} \sin \left[2 \pi \theta_{1}(t)\right] & (\bmod 1) \\
p_{2}(t+1)=p_{2}(t)+\frac{a_{2}}{2 \pi} \sin \left[2 \pi \theta_{2}(t)\right] & (\bmod 1) \\
\theta_{1}(t+1)=p_{1}(t+1)+\theta_{1}(t)+b p_{2}(t+1) & (\bmod 1) \\
\theta_{2}(t+1)=p_{2}(t+1)+\theta_{2}(t)+b p_{1}(t+1) & (\bmod 1)
\end{array}
$$

where $a_{1}, a_{2}, b \in \mathbb{R}, \quad t=0,1, \ldots, b$ is the coupling constant, $\theta_{i}$ may be regarded as an angular variable, and $p_{i}$ as an angular momentum $(i=1,2)$. In the 
(a)

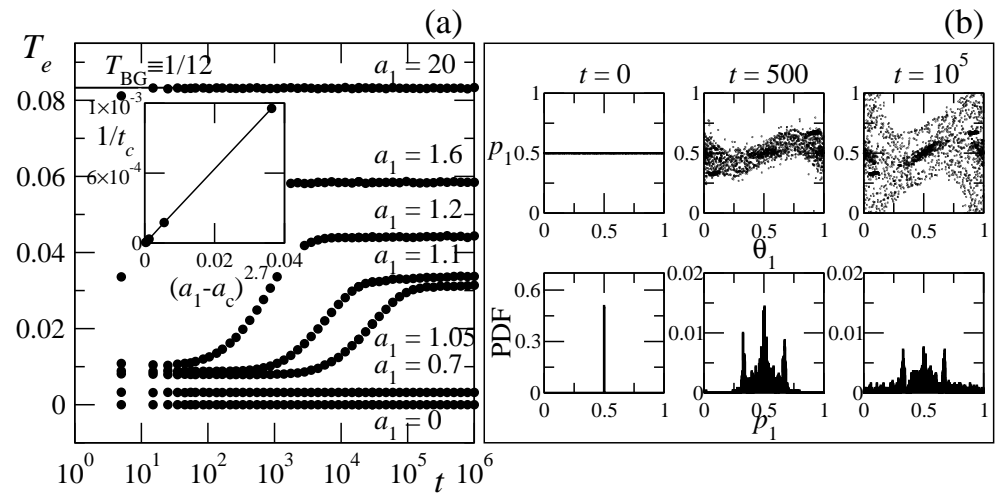

Fig. 1. Case (A) 'Ensemble' QSSs in the standard map. (a) $T_{e}(t)$ for typical values of $a_{1}$. We start with 'water bag' initial conditions $\left(M=2500\right.$ points in $0 \leq \theta_{1} \leq 1$, $p_{1}=0.5 \pm 510^{-4}$ ). Inset: Inverse crossover time $t_{c}$ (inflection point between the QSS and the BG regime) vs. $1 /\left(a_{1}-a_{c}\right)^{2.7}$. (b) Time evolution of the ensemble in (a) for $a_{1}=1.1$ (first row) and PDF of its angular momentum (second row). $t=0$ : initial conditions; $t=t_{1}=500$ : the ensemble is mostly restricted by cantori; $t=t_{2}=10^{5}$ : the ensemble is confined inside KAM-tori. See [4] for further details.

following we will take into account two cases: (A) for $b=0$ we consider only coordinates 1 , that is, the 2-dimensional standard map; (B) we fix $b=2$ and $a_{1}=a_{2} \equiv \tilde{a}$ in order to analyze the 4-dimensional map (see [4] for details). The standard map is integrable when $a_{1}=0$, while chaoticity rapidly increases with $\left|a_{1}\right|$; we also remind that for positive $a_{1}$ smaller than $a_{c}=0.971635406 \ldots$ the (macroscopic) chaotic sea is disconnected, because of the presence of invariant trajectories, called KAM-tori, that span the whole interval $\theta \in[0,1]$.

With some similarity with the so-called 'water bag' initial data that produce QSSs in the HMF model [1], we consider at $t=0$ a statistical ensemble of $M$ copies of the map with arbitrary $\theta_{i}$ and $p_{i}$ randomly distributed inside small regions of the interval $[0,1]$. Usually, in the connection between statistical mechanics and dynamics for systems with diagonal kinetic matrix and zero average momentum, the temperature is set proportional to the average square momentum per particle (see, e.g., [9]). As we address situations with nonzero 'bulk' motion, the analogous concept, here called dynamical temperature, can be defined as the (specific) variance of the total angular momentum:

$$
T_{e}(t) \equiv \frac{1}{d / 2} \sum_{i=1}^{d / 2}\left(\left\langle p_{i}^{2}(t)\right\rangle-\left\langle p_{i}(t)\right\rangle^{2}\right), \quad(\mathbf{A}): d=2, \quad(\mathbf{B}): d=4
$$

where \langle\rangle means ensemble average. The qualification dynamical is used since this definition purely descends from dynamics and not from a thermal contact with a thermometer. The temperature associated with the uniform distribution in the entire phase space can be called $B G$ temperature and it is given, for both cases, by $T_{\mathrm{BG}} \equiv \frac{1}{d / 2} \sum_{i=1}^{d / 2}\left[\int_{0}^{1} d p_{i} p_{i}^{2}-\left(\int_{0}^{1} d p_{i} p_{i}\right)^{2}\right]=1 / 12 \simeq 0.083$. 


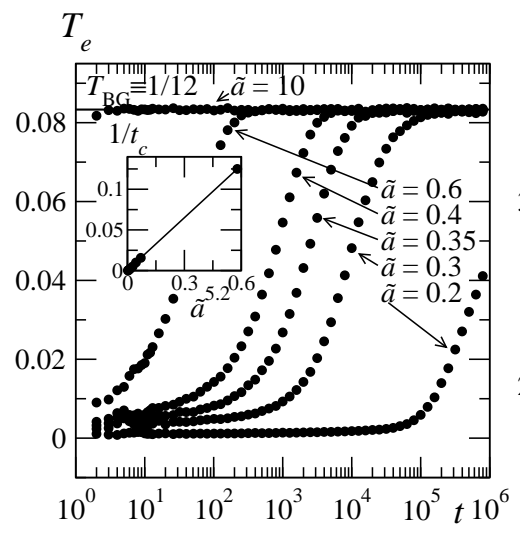

(a)

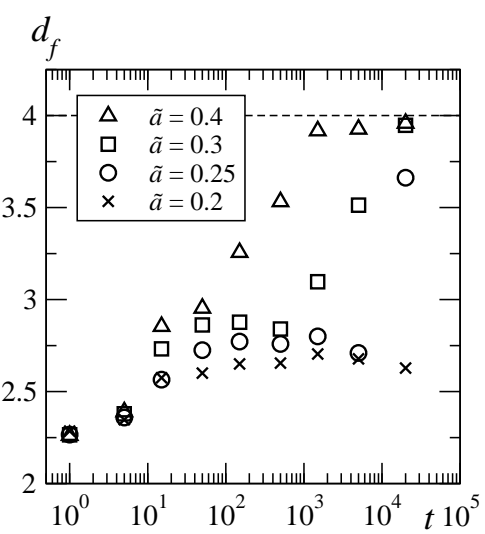

(b)

Fig. 2. Case (B) 'Ensemble' QSSs in two coupled standard maps. (a) $T_{e}(t)$ for $b=2$ and typical values of $\tilde{a}$. We start with 'water bag' initial conditions $(M=1296$ points with $0 \leq \theta_{1}, \theta_{2} \leq 1$, and $p_{1}, p_{2}=0.25 \pm 510^{-3}$ ). Inset: Inverse crossover time $t_{c}$ vs. $1 / \tilde{a}^{5.2}$. (b) Time evolution of the fractal dimension of a single initial ensemble in the same setup of (a). See [4] for further details.

For large values of $\left|a_{1}\right|$ (case (A)) or $|\tilde{a}|$ (case (B)) the system is typically mixing and it rapidly relaxes the dynamical temperature to $T_{B G}$. Reduction of $\left|a_{1}\right|$ or $|\tilde{a}|$ causes the formation of islands, barriers and partial barriers in phase space. The latter are responsible for the appearance of the QSSs, since they confine the ensemble inside a limited volume during a certain time. If the initial data are defined inside a singly connected volume that does not contain islands and if the the available portion of phase space for the final relaxation of the ensemble is such that its projection over the $\left(p_{1}, p_{2}\right)$-plane $\left(p_{1}\right.$-axis in case (A)) is uniform, the final temperature coincides with $T_{B G}$. We remark that this is possible only if the dimension of the map is larger than two [4]. Fig. 1 and Fig. 2 display this behavior for cases (A) and (B) respectively. It is also important to notice that a nontrivial fractal dimension characterizes the QSSs, while, once the cross over to $T_{B G}$ is performed, the dimension of the ensemble coincides with that of the phase space (see Fig. 2 (b)). This indicates a strong violation, during the QSSs, of the equal-a-priori probability postulate on which BG statistical mechanics relies.

\section{$3 \quad$ QSSs using time averages}

Given a dynamical function $f$ that takes different values $f\left(x_{0}, t\right)$ along a single trajectory started in the phase space point $x_{0}$, we define its time average $\bar{f}\left(x_{0}, \bar{t}, t_{o}\right)$ at time $t=\bar{t}$ during the observation time $t_{o} \equiv t_{f}-t_{i}$ as

$$
\bar{f}\left(x_{0}, \bar{t}, t_{o}\right) \equiv \frac{\sum_{t=t_{i}}^{t=t_{f}} f\left(x_{0}, t\right)}{t_{f}-t_{i}},
$$




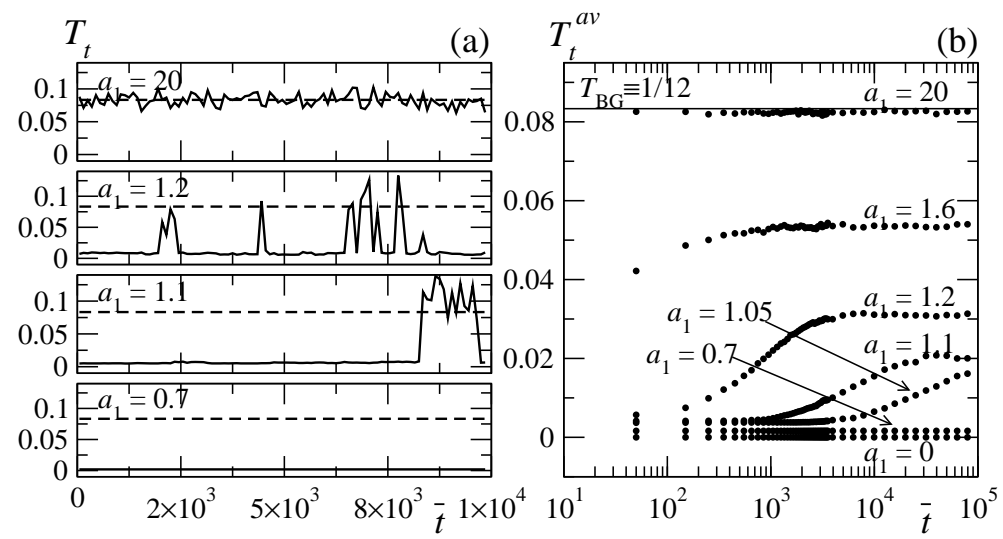

Fig. 3. Case (A) 'Time' QSSs in the standard map. (a) $T_{t}(\bar{t})$ for typical values of $a_{1}$. Evolution for a single orbit started in $x_{0}=(0.3,0.5)$, with observation interval $t_{o}=10^{2}$. Dashed lines indicate $T_{B G}$. (b) Averages over $10^{5}$ realizations of the kind in (a), with initial data inside $0 \leq \theta_{1} \leq 1: p_{1}=0.5 \pm 510^{-4}$.

where $t_{i}$ and $t_{f}$ are respectively the initial and final observation time, and $\bar{t} \equiv t_{i}+t_{o} / 2$. This definition mimics the action of a detector testing the behavior of the system over a portion of a particular trajectory. Within this context, the definition of the dynamical temperature is reproduced as

$$
T_{t}\left(x_{0}, \bar{t}, t_{o}\right) \equiv \frac{1}{d / 2} \sum_{i=1}^{d / 2}\left(\overline{p_{i}^{2}}-\bar{p}_{i}^{2}\right), \quad(\mathbf{A}): d=2, \quad(\mathbf{B}): d=4
$$

For uniform distributions of momenta over the whole phase space during the observation interval $t_{o}$ we obtain once again $T_{t}=T_{B G}$.

When the system is sufficiently chaotic, as expected, $T_{t}$ oscillates around $T_{B G}$. On the other hand, if $\left|a_{1}\right|$ (case (A)) or $|\tilde{a}|$ (case (B)) are diminished, $T_{t}$ exhibits, instead of the two plateaux structure, an intermittent behavior. This indicates the presence of sequences of partial barriers along the phase space trajectory, that alternate rapid and slow diffusion processes. Notice that $T_{t}$ can jump to larger values than $T_{B G}$ (i.e., the variance of the time-distribution of total momentum can become larger than that of a uniform distribution). Fig. 3(a) and 4(a) show this behavior for cases (A) and (B) respectively, for typical orbits.

Another interesting quantity is the average value of $T_{t}\left(x_{0}, \bar{t}, t_{o}\right)$ calculated for different orbits $x_{0}: T_{t}^{a v}\left(\bar{t}, t_{o}\right) \equiv\left\langle T_{t}\right\rangle_{x_{0}}$. Of course, $T_{t}^{a v}$ coincides with $T_{e}$ for $t_{o}=1$, while for $t_{0}>1$ it represents the average result of different measurements and it is a typical observable calculated in the ergodicity analysis (see. e.g., [9]). Fig. 3(b) and 5(a) display that in this way we obtain a behavior qualitatively equivalent to the ensemble averages of Fig. 1(a) and Fig. 2(a), but typically with lower temperatures. Interestingly enough, 5(b) seems to indicate a tendency for $T_{t}^{a v}$ to approach $T_{e}$, as $t_{o}$ increases. 


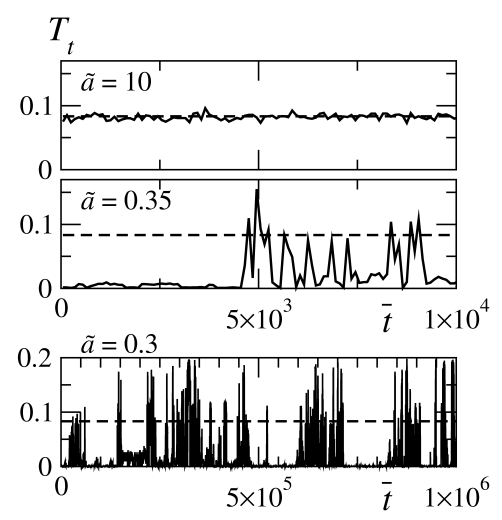

(a)

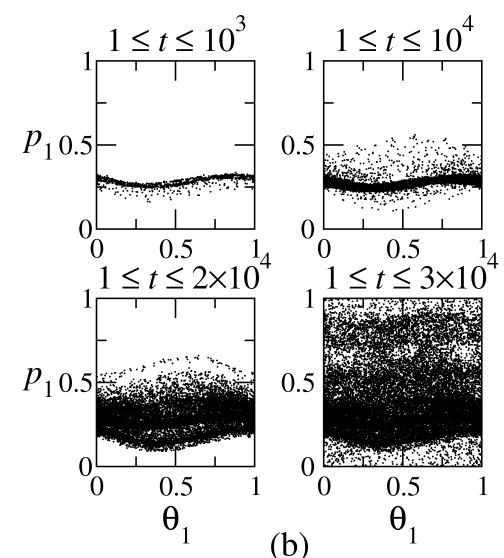

(b)

Fig. 4. Case (B). (a) $T_{t}(\bar{t})$ for $b=2$ and typical values of $\tilde{a}$. Evolution for a single orbit started in $x_{0}=(0.5,0.25,0.8,0.25)$, with observation interval $t_{o}=10^{2}$. Dashed lines indicate $T_{B G}$. (b) Phase space analysis of the trajectory in (a) for $\tilde{a}=0.3$. Dots represent the projection of the orbit on the plane $\left(\theta_{1}, p_{1}\right)$.

\section{Conclusions}

We have addressed a simple connection between nonlinear dynamical systems and thermostatistics. Using well known results in symplectic maps (see, e.g., $[6,7]$ ), we pointed out the presence of QSSs with remarkable similarities with analogous ones detected in the HMF model [1]. Common features include the presence of an anomalous temperature-plateau in correspondence of particular values of a control parameter $\left(a_{1}\right.$ or $\tilde{a}$ for the maps, the specific energy for the HMF). QSSs appear in both cases for particular classes of initial data and after a certain amount of time a cross over to equilibrium is observed. As the number $N$ of coupled elements goes to infinity, the duration of the QSS diverges (see [1] for the HMF and [5] for the maps), opening the possibility for this anomalous effect to be physically relevant if the thermodynamic limit is taken before the infinite-time limit. All these similarities endow with renewed interest the analysis of dynamical mechanisms of symplectic maps. For example, an important result is that during the QSSs of low-dimensional symplectic maps the phase space occupation exhibits evidences of fractalization that disappear once equilibrium is attained.

The 'core' of equilibrium statistical mechanics consists in the elimination of the time variable as can be justified for example by the ergodic hypothesis. In this paper, working with low-dimensional symplectic maps, we have illustrated that time and ensemble averages, due to the complexity of phase space, originate different behaviors. A single time average of the dynamical temperature, a variable that was introduced in [4] in order to enable a natural comparison with many-body Hamiltonian systems, displays an intermittent behavior. In contrast, averaging many of these histories with a large enough observation time we observed a two-plateaux structure qualitatively similar to the one 


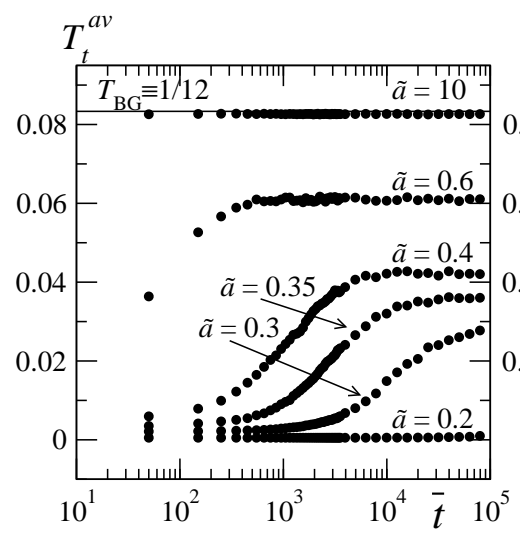

(a)

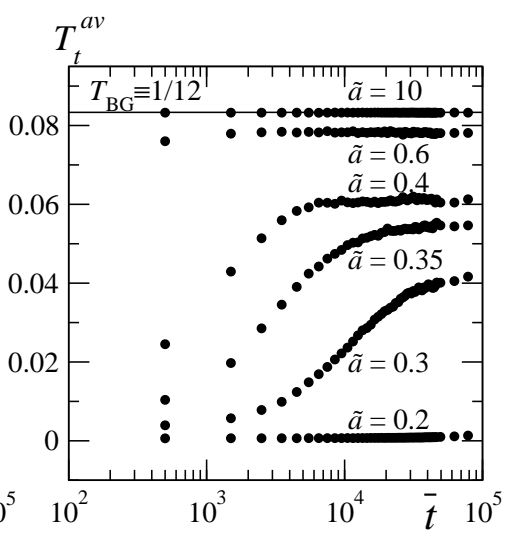

(b)

Fig. 5. Case (B) 'Time' QSSs in two coupled standard maps. $T_{t}^{a v}(\bar{t})$ obtained averaging $10^{5}$ realizations of the kind in Fig. 4 , with initial data inside $0 \leq \theta_{1}, \theta_{2} \leq 1$, $p_{1}, p_{2}=0.25 \pm 510^{-3}$. (a) The observation interval is $t_{o}=10^{2}$. (b) The observation interval is $t_{o}=10^{3}$.

obtained with ensemble averages.

Acknowledgments: I acknowledge C. Tsallis, A. Robledo, A. Rapisarda, and E. Brigatti for useful discussions and comments, and M. Zamberlan for tireless encouragement. I have benefitted from partial support by FAPERJ, CNPq and PRONEX (Brazilian agencies).

\section{References}

[1] See, e.g., C. Tsallis, A. Rapisarda, V. Latora and F. Baldovin, in Dynamics and Thermodynamics of Systems with Long-Range Interactions, eds T. Dauxois, S. Ruffo, E. Arimondo and M. Wilkens Lecture Notes in Physics 602, 140 (Springer, Berlin, 2002) and references therein.

[2] M.A. Montemurro, F. Tamarit and C. Anteneodo, Phys. Rev. E 67, 031106 (2003).

[3] A. Pluchino, V. Latora and A. Rapisarda, cond-mat/0306374.

[4] F. Baldovin, E. Brigatti and C. Tsallis, Phys. Lett. A 320, 254 (2004).

[5] F. Baldovin, L.G. Moyano, A.P. Majtey, A. Robledo and C. Tsallis, cond-mat/0312407.

[6] R.S. Mackay, J.D. Meiss and I.C. Percival, Physica D 13, 55 (1984).

[7] K. Kaneko and T. Konishi, Physica D 71, 146 (1994).

[8] See, e.g., E. Ott, Chaos in dynamical systems (Cambridge University Press, 1993) and references therein.

[9] R. Livi, M. Pettini, S. Ruffo and A. Vulpiani, J. Stat. Phys. 48, 539 (1987). 